\title{
A Method of Locating the Dehiscence during Middle Fossa Approach for Superior Semicircular Canal Dehiscence Surgery
}

\author{
Joel S. Beckett ${ }^{1}$ Lawrance K. Chung ${ }^{1}$ Carlito Lagman \\ Bilwaj Gaonkar ${ }^{1}$ Quinton Gopen ${ }^{2}$ Isaac Yang ${ }^{1,2,3,4}$ \\ ${ }^{1}$ Department of Neurosurgery, University of California, Los Angeles,
Los Angeles, California, United States
${ }^{2}$ Department of Head and Neck Surgery, University of California,
Los Angeles, Los Angeles, California, United States
${ }^{3}$ Department of Radiation Oncology, University of California,
Los Angeles, Los Angeles, California, United States
${ }^{4}$ Jonsson Comprehensive Cancer Center, University of California,
Los Angeles, Los Angeles, California, United States
}

\author{
Brittany L. Voth ${ }^{1}$
}

Cheng Hao Jacky Chen ${ }^{1}$

Address for correspondence Isaac Yang, MD, Department of Neurosurgery, University of California, Los Angeles, 300 Stein Plaza, suite 562, Los Angeles, CA 90095-6901, United States (e-mail: iyang@mednet.ucla.edu).

J Neurol Surg B 2017;78:353-358.

\begin{abstract}
Objectives Superior semicircular canal dehiscence $(S S C D)$ results from a defect in the middle cranial fossa floor. One challenge during SSCD repair is the lack of a consistent landmark. This study proposes a reference point above the external auditory canal at the level of the zygoma as the inferior craniectomy edge during surgery.

Design This is a retrospective review of patients with SSCD.

Setting/Participants A total of 72 cases of SSCD in 60 patients were repaired via a middle fossa approach at a single institution.

Main Outcome Measures The distance from the proposed reference point to the dehiscence was statistically analyzed using Shapiro-Wilk's goodness-of-fit test and Student's t-test.

Results Average distance for all patients was $28.84 \pm 2.22 \mathrm{~mm}$ (range: $22.96-33.43$ ). Average distance for females was $29.08 \mathrm{~mm}$ (range: 24.56-33.43) versus $28.26 \mathrm{~mm}$ (range: $22.96-32.36$ ) for males. There was no difference in distance by sex $(p=0.174$ ).

\section{Keywords}

- superior semicircular canal dehiscence

- syndrome

- distance

- measurement The distance measurements followed a normal distribution with $95 \%$ of the patients between 24.49 and $33.10 \mathrm{~mm}$.

Conclusion This study analyzed a potential reference point during a middle fossa approach for SSCD surgery. The distance from this reference point to the SSCD was found to be consistent and may serve as a readily identifiable landmark in localizing the dehiscence.
\end{abstract}

\section{Introduction}

Superior semicircular canal dehiscence (SSCD) describes a relatively rare syndrome of auditory and vestibular symptoms due to a bony defect between the middle cranial fossa and the superior semicircular canal. ${ }^{1}$ The bony defect creates a third window in the inner ear, altering the fluid dynamics within the auditory and vestibular systems. ${ }^{2}$ The ensuing constellation of sound-induced vertigo, pressure-induced vertigo, hearing loss, pulsatile tinnitus, aural fullness, and autophony describe the symptoms of SSCD. ${ }^{3-6}$ Patients with signs and symptoms consistent with SSCD are most often

(c) 2017 Georg Thieme Verlag KG Stuttgart · New York
DOI https://doi.org/ 10.1055/s-0037-1601886. ISSN 2193-6331. 
diagnosed through high-resolution computed tomography (CT) scans of the temporal bone. ${ }^{7,8}$ Once confirmed, symptomatic SSCD refractive to nonsurgical management may be corrected via surgery by plugging or resurfacing the superior semicircular canal to eliminate the third window effect.

Surgical correction of SSCD has traditionally been accomplished through a middle cranial fossa approach. Although a middle fossa approach has excellent clinical outcomes, it is not without its challenges. ${ }^{9-12}$ One such challenge has been the lack of a consistent and readily identifiable landmark on the cranial floor. By convention, the arcuate eminence is believed to represent the protrusion of the superior semicircular canal into the middle fossa floor. However, both anatomical and radiological studies have shown that this relationship may not be entirely accurate. ${ }^{13-18}$ Even in situations where the anatomical relationship is correct, identifying the arcuate eminence may be challenging as patients with SSCD potentially have a flattened or altered arcuate eminence around the dehiscent area. Hence, the location of an easily identifiable and consistent reference point is needed.

In performing a middle fossa approach for SSCD repair, the senior authors of this study (Q.G. and I.Y.) standardized the location of the craniectomy site. Incidentally, the authors observed that the location of the canal dehiscence was $\sim 3 \mathrm{~cm}$ from this location in a majority of patients. A similar study was completed by Patel et al. who utilized the external auditory canal as a possible reference point. ${ }^{19}$ This study builds upon that prior study by using a better defined craniectomy site and a different method of distance determination. The objective of the current study is to analyze whether the craniectomy site used by the senior authors may serve as a consistent landmark among a larger series of SSCD patients. The establishment of a surgical reference point could potentially decrease the risk associated with a middle fossa approach through an easily identifiable landmark that may be used during SSCD repair.

\section{Methods}

A retrospective chart review was conducted to identify all patients with radiologically confirmed SSCD at the Ronald Reagan UCLA Medical Center who underwent surgical repair via a middle fossa approach. Baseline patient characteristics were then extracted from the electronic medical record system. Dedicated temporal bone CT scans ( $0.6 \mathrm{~mm}$ slice) for all patients were deidentified and imported into ITKSNAP (v.3.4.0) software. The initial measurements were performed by the third author (C.L.) and then verified by the first author (J.S.B.). This study was approved by the University of California, Los Angeles Institutional Review Board (IRB\#15-000252).

\section{Identification of Reference Point}

Intraoperatively, the preferred craniectomy site was determined by palpating and exposing the zygomatic root, and then identifying the superior rim of the bony external auditory canal. A parallel line was then drawn at the superior

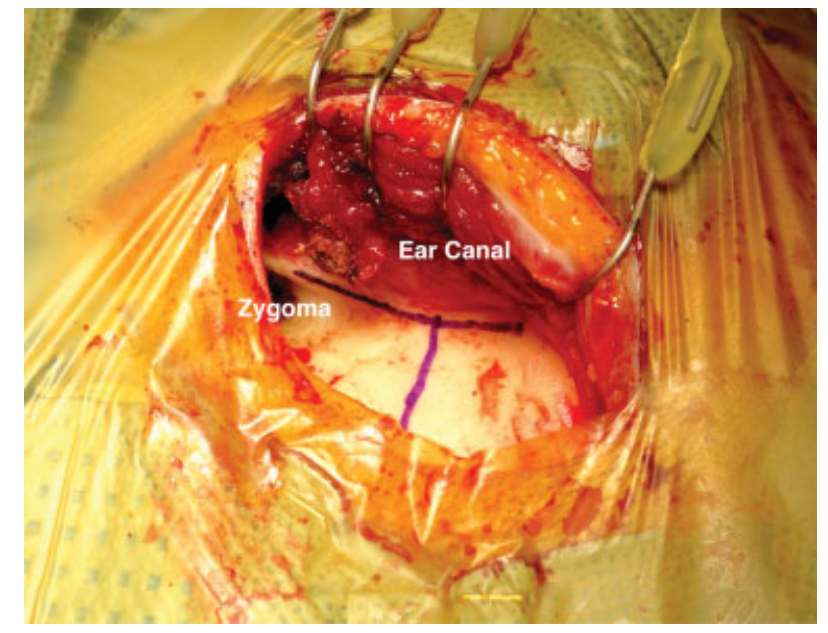

Fig. 1 An intraoperative photograph demonstrating the location of the craniectomy site as identified by the intersection of a parallel line drawn from the superior border of the zygomatic root and a perpendicular line drawn from the midpoint of the external auditory canal.

border of the zygomatic root, while a perpendicular line was extended rostrally from the midpoint of the external auditory canal (-Fig. 1). The intersection of these two lines was then used as the location of the inferior border of a circular craniectomy $\sim 1.5$ to $2 \mathrm{~cm}$ in diameter.

\section{Distance Determination}

For our image processing analysis, the craniectomy site was found radiographically on preoperative scans. First, axial images were used to estimate the center of the external auditory meatus. Image slices were then advanced rostrally until passing the superior most border of the posterior root of the zygoma, where a point was then placed on the outer cortex of the squamous temporal bone to denote the craniectomy site. The accuracy in delineating the craniectomy site was confirmed by referencing the postoperative CT in several patients. The area of dehiscence was then identified in a similar manner by placing a point in the superior most aspect of the dehiscence as denoted by bony lucency. The dehiscent area was then confirmed by referencing the axial, coronal, and sagittal planes. The distance between the reference point and dehiscence was then calculated using an automated Python script. From this reference point, the dehiscence was located approximately perpendicular from the temporal bone. - Fig. 2 shows a three-dimensional reconstruction of a patient skull with SSCD and the aforementioned method of identifying the craniectomy site and distance measurement.

\section{Statistical Analysis}

Statistical analyses were performed using SPSS v.22.0 (IBM Corporation) with $p$-values less than 0.05 considered statistically significant. Standard SPSS procedures were used to determine the means and frequency count for continuous variables. A Shapiro-Wilk's goodness-of-fit test was used 

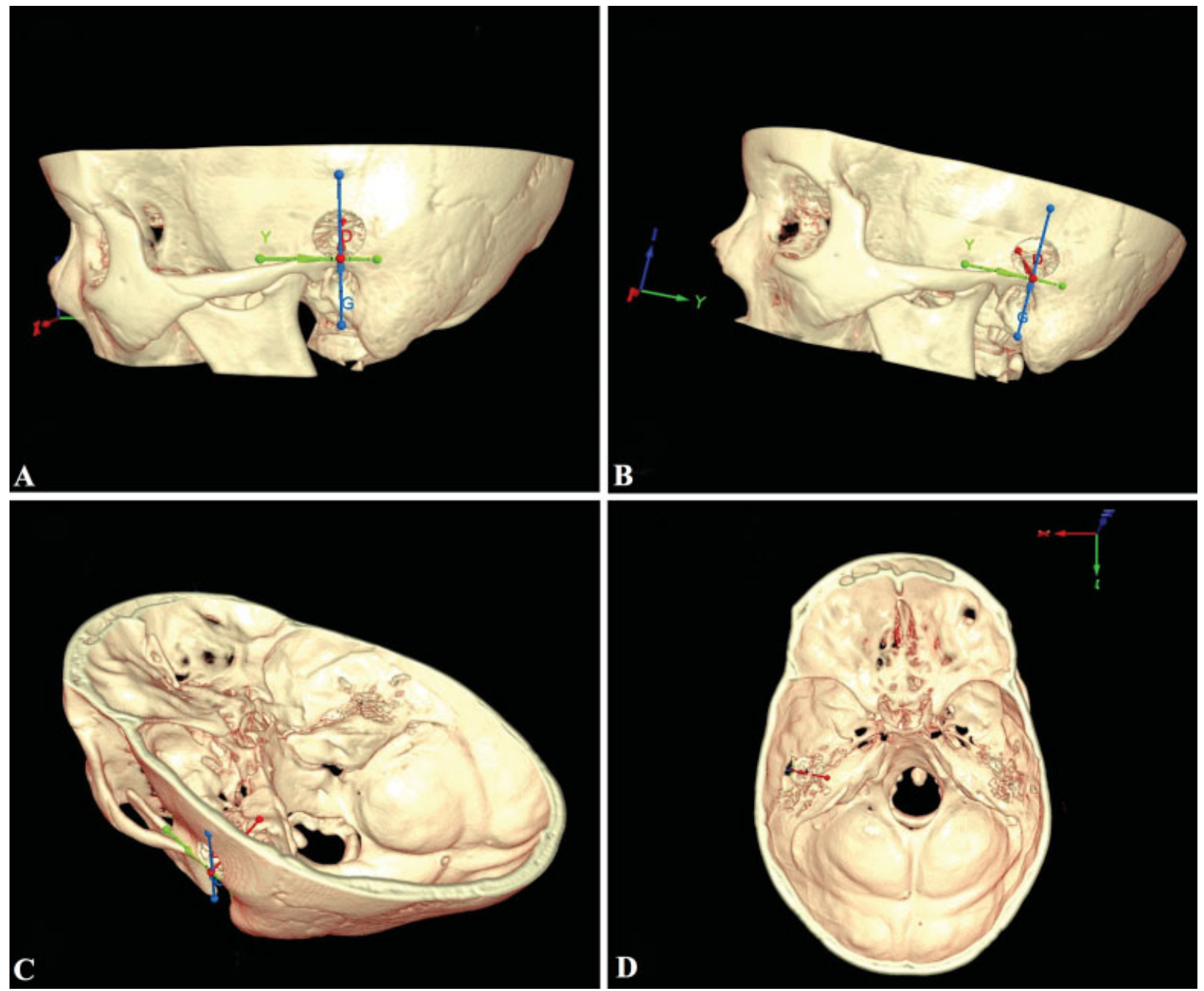

Fig. 2 A three-dimensional CT reconstruction of a patient skull with SSCD demonstrating the craniectomy site as the intersection of a parallel line drawn from the superior border of the zygomatic root (Y: Yang line) and a perpendicular line drawn from the midpoint of the external auditory canal (G: Gopen line), with the measurement (D: distance) to the dehiscence. Panels A to D show this relationship in multiple views. CT, computed tomography; SSCD, superior semicircular canal dehiscence.

to evaluate whether the distance measurements followed a normal distribution. Differences in distance by sex were evaluated using a Student's $t$-test after testing for equality of variances. Since variances were unequal in our data, Welch-Satterthwaite's $p$-values were used.

\section{Results}

A total of 72 cases of SSCD were identified in 60 patients between August 2013 and December 2015. Average age was 50.8 years (range: $21-84$ years). Females comprised $66.7 \%$ $(n=40)$ and males comprised $33.3 \%(n=20)$ of the study, with a female-to-male ratio of $2: 1$.

Average distance between the reference point and dehiscence for all patients was $28.84 \pm 2.22 \mathrm{~mm}$ (range: 22.96 $33.43,95 \%$ confidence interval [CI]: 28.32-29.36). Average distance for females was $29.08 \mathrm{~mm}$ (range: 24.56-33.43) versus $28.26 \mathrm{~mm}$ (range: $22.96-32.36$ ) for males. The difference in distance by sex was not statistically significant $(p=0.174)$. The distance measurements followed a normal distribution (Shapiro-Wilk statistic $=0.988 ; p=0.722$ ). Given the normal distribution, $68 \%$ of the patients had distances between 26.63 and $31.05 \mathrm{~mm}$ and $95 \%$ of the patients had distances between 24.49 and $33.10 \mathrm{~mm}$ from the site of the craniectomy.

\section{Discussion}

In this study, the authors analyzed the potential of a reference point in locating the area of dehiscence in patients undergoing surgical repair of SSCD via a middle fossa approach. The authors performed radiological measurements of 72 cases of SSCD to characterize this easily identifiable reference point as the location of the craniectomy, and measured its distance to the dehiscent defect. In doing so, the location of the dehiscence was found to be nearly $29 \mathrm{~mm}$ from the reference point, with $95 \%$ of the patients falling between $\sim 25$ and $33 \mathrm{~mm}$. 
The need for a reliable reference point during a middle fossa approach for SSCD repair is due to the inconsistent relationship between the arcuate eminence and superior semicircular canal, and the variable location of the dehiscence along the length of the canal. Without a reliable reference point, intimate knowledge of temporal bone anatomy is required whenever anatomical variations are encountered during surgery. Numerous studies have challenged the traditional teaching that the arcuate eminence represents the projection of the superior semicircular canal on the middle fossa floor. In a study of 100 temporal bone CT images, Faure et al. found that the arcuate eminence corresponded with the superior semicircular canal only $37 \%$ of the time. ${ }^{16}$ Other studies have reported rates as low as 17 to $20 \%$ of finding this anatomical relationship. ${ }^{14,20}$ The arcuate eminence has even been reported to be absent in 15 to $30 \%$ of temporal bone specimens. ${ }^{16,21}$ Furthermore, the dehiscence may have a variable location on the superior semicircular canal itself. Lookabaugh et al. studied 392 temporal bones in 202 patients with symptomatic SSCD and found that the most common location of the dehiscence was the arcuate eminence (59.2\%), followed by the medial downslope of the semicircular canal (28.8\%), then the lateral upslope of the semicircular canal (7.6\%). ${ }^{22}$ The same study also reported that the arcuate eminence was found an average of $26.41 \mathrm{~mm}$ (range: 17.6-38.5) from the outer cortex of the temporal bone.

Studies that qualify the location of the arcuate eminence may prove useful, as current operative methodology relies heavily on locating the arcuate eminence as a landmark. Once the arcuate eminence has been identified, the surgeon can then use distance measurements to verify its location and look for the nearby bony defect. However, as previously described, such a method may be problematic in several patients. One of the main limitations is that this approach relies on a landmark encountered intraoperatively, rather than preoperatively. To overcome this challenge, Patel et al. suggested using the external auditory canal as the reference point. ${ }^{19}$ The authors measured the horizontal distance from the squama temporalis to the dehiscence in 151 temporal bones and found that the average distance to the SSCD was $25.9 \mathrm{~mm}$ (range: $17.8-33.2$ ). This method allows for an easily identifiable reference point prior to dural elevation and can be generalized for all patients, regardless of the relationship between the superior semicircular canal and arcuate eminence. However, such a measurement may differ intraoperatively depending on whether the inferior edge of the craniotomy/craniectomy is flush with the middle fossa floor or whether the radiological measurements were calculated in the true coronal plane without skewness from head misalignment. To overcome this limitation, the method used in this current study measures the length of the distance through several planes. This method provides a closer approximation to measurements obtained intraoperatively as a ruler used during surgery would measure the distance from the craniectomy edge to the dehiscence directly, rather than in just the axial plane across the temporal floor.
The authors of this study used a consistent craniectomy site as identified by the intersection of two lines drawn from the superior border of the zygomatic root and the midpoint of the external auditory canal. By doing so, we introduce an easily identifiable reference point that is immediately visible at the start of the surgery. Using a standardized craniectomy site may help provide consistent results between distances measured radiologically and intraoperatively. As such, our average distance demonstrates a narrow 95\% CI and a small standard deviation. Despite differences in anatomical relationships and variable SSCD locations, $95 \%$ of our patients had a distance measurement that varied by less than $1 \mathrm{~cm}$ from each other. Since SSCD patients are diagnosed via CT scans of the temporal bone, this distance measurement can be readily calculated for all patients to aid in the localization of the dehiscence during surgery. The findings of this study have, in part, allowed the senior authors to modify the traditional middle fossa craniotomy for SSCD repair into a minimally invasive keyhole craniectomy instead. ${ }^{23}$ Other benefits may include increased surgical efficacy by reducing the risk of resurfacing or plugging the wrong defect and decreased operative time; however, such benefits will need to be confirmed in future studies.

\section{Limitations}

The measurements within this study were all obtained retrospectively via $\mathrm{CT}$ images of temporal bones in patients who underwent SSCD repair. None of these measurements was confirmed intraoperatively. However, given the authors' experience of using this reference point for the past 3 years, we have no reason to believe that the findings of this study cannot be successfully applied in the clinical setting. For a future study, obtaining distance measurements intraoperatively would provide confirmation of our results. Obtaining accurate and reliable distance measurements from CT images requires high-quality and consistent scans. All images used in this study were acquired with uniform scanning techniques at a single institution. In addition, motion artifacts or misalignment may affect the accuracy of results. However, we did not encounter these issues in our study cohort, but caution should be taken if these issues were encountered.

\section{Conclusion}

A consistent and reliable reference point for SSCD surgery may help address some of the challenges associated with a middle fossa approach, namely, the inconsistent relationship between the arcuate eminence and superior semicircular canal. This present study analyzed a reference point located at the level of the zygoma and the midpoint of the external auditory canal as the inferior border of the craniectomy. The distance from this reference point to the SSCD was found to be $\sim 29 \mathrm{~mm}$, with $95 \%$ of the patients falling within $4 \mathrm{~mm}$ of this measurement. This readily identifiable and consistent reference point may be used during SSCD surgery to aid in localizing the dehiscence. 


\section{Conflict of Interest}

The authors report no conflict of interest concerning the materials or methods used in this study or the findings specified in this article.

\section{Acknowledgments}

We thank Patrick O'Neal for his assistance in creating the three-dimensional reconstruction images used in this study. Lawrance K. Chung was partially supported by an AMA Foundation Seed Grant, an A AA Carolyn L. Kuckein Student Research Fellowship, and Gurtin Skull Base Research Fellowship. Carlito Lagman was partially supported by a Gurtin Skull Base Research Fellowship. Isaac Yang was partially supported by a Visionary Fund Grant, an Eli and Edythe Broad Center of Regenerative Medicine and Stem Cell Research UCLA Scholars in Translational Medicine Program Award, the Jason Dessel Memorial Seed Grant, the UCLA Honberger Endowment Brain Tumor Research Seed Grant, and the STOP CANCER Research Career Development Award.

\section{References}

1 Minor LB, Solomon D, Zinreich JS, Zee DS. Sound- and/or pressureinduced vertigo due to bone dehiscence of the superior semicircular canal. Arch Otolaryngol Head Neck Surg 1998;124(3): 249-258

2 Merchant SN, Rosowski JJ, McKenna MJ. Superior semicircular canal dehiscence mimicking otosclerotic hearing loss. Adv Otorhinolaryngol 2007;65:137-145

3 Minor LB. Clinical manifestations of superior semicircular canal dehiscence. Laryngoscope 2005;115(10):1717-1727

4 Chien W, Ravicz ME, Rosowski JJ, Merchant SN. Measurements of human middle- and inner-ear mechanics with dehiscence of the superior semicircular canal. Otol Neurotol 2007;28(2):250-257

5 Ramsey MJ, McKenna MJ, Barker FG II. Superior semicircular canal dehiscence syndrome. Case report. J Neurosurg 2004;100(1): 123-124

6 Minor LB, Carey JP, Cremer PD, Lustig LR, Streubel SO, Ruckenstein MJ. Dehiscence of bone overlying the superior canal as a cause of apparent conductive hearing loss. Otol Neurotol 2003;24(2): 270-278

7 Belden CJ, Weg N, Minor LB, Zinreich SJ. CT evaluation of bone dehiscence of the superior semicircular canal as a cause of soundand/or pressure-induced vertigo. Radiology 2003;226(2):337-343
8 Cloutier JF, Bélair M, Saliba I. Superior semicircular canal dehiscence: positive predictive value of high-resolution CT scanning. Eur Arch Otorhinolaryngol 2008;265(12):1455-1460

9 Chung LK, Ung N, Spasic M, et al. Clinical outcomes of middle fossa craniotomy for superior semicircular canal dehiscence repair. J Neurosurg 2016;125(5):1187-1193

10 Goddard JC, Wilkinson EP. Outcomes following semicircular canal plugging. Otolaryngol Head Neck Surg 2014;151(3): 478-483

11 Mikulec AA, Poe DS, McKenna MJ. Operative management of superior semicircular canal dehiscence. Laryngoscope 2005; 115(3):501-507

12 Niesten ME, McKenna MJ, Grolman W, Lee DJ. Clinical factors associated with prolonged recovery after superior canal dehiscence surgery. Otol Neurotol 2012;33(5):824-831

13 Tsunoda A, Kimura Y, Sumi T, Komatsuzaki A, Sato T. The arcuate eminence is not a protrusion of the superior semi-circular canal but a trace of sulcus on the temporal lobe. J Laryngol Otol 2000; 114(5):339-344

14 Seo Y, Ito T, Sasaki T, Nakagawara J, Nakamura H. Assessment of the anatomical relationship between the arcuate eminence and superior semicircular canal by computed tomography. Neurol Med Chir (Tokyo) 2007;47(8):335-339, discussion 339-340

15 Kartush JM, Kemink JL, Graham MD. The arcuate eminence. Topographic orientation in middle cranial fossa surgery. Ann Otol Rhinol Laryngol 1985;94(1 Pt 1):25-28

16 Faure A, Masse H, Gayet-Delacroix M, et al. What is the arcuate eminence? Surg Radiol Anat 2003;25(2):99-104

17 Bulsara KR, Leveque JC, Gray L, Fukushima T, Friedman AH, Villavicencio AT. Three-dimensional computed tomographic analysis of the relationship between the arcuate eminence and the superior semicircular canal. Neurosurgery 2006;59(1, Suppl 1): ONS7-ONS12, discussion ONS7-ONS12

18 Arìstegui M, Cokkeser Y, Saleh E, et al. Surgical anatomy of the extended middle cranial fossa approach. Skull Base Surg 1994; 4(4):181-188

19 Patel NS, Hunter JB, O'Connell BP, Wanna GB, Carlson ML. An easy and reliable ethod to locate the dehiscence during middle fossa superior canal dehiscence surgery: it is a (C)inch. Otol Neurotol 2016;37(8):1092-1095

20 Djalilian HR, Thakkar KH, Hamidi S, Benson AG, Mafee MF. A study of middle cranial fossa anatomy and anatomic variations. Ear Nose Throat J 2007;86(8):474, 476-481

21 Sennaroglu L, Slattery WH III. Petrous anatomy for middle fossa approach. Laryngoscope 2003;113(2):332-342

22 Lookabaugh S, Kelly HR, Carter MS, et al. Radiologic classification of superior canal dehiscence: implications for surgical repair. Otol Neurotol 2015;36(1):118-125

23 Trieu V, Pelargos PE, Spasic M, et al. Minimally invasive middle fossa keyhole craniectomy for repair of superior semicircular canal dehiscence. Oper Neurosurg 2017. doi: 10.1093/ons/opw046 
